\title{
Correction to: advanced olfactory neuroblastoma in a teenager: a clinical case and short review of literature
}

\author{
Gino Amleto Pacino ${ }^{1}$. Salvatore Cocuzza ${ }^{2} \cdot$ Antonino Maniaci $^{2} \cdot$ Maria Cristina Da Mosto $^{3} \cdot$ Piero Pavone $^{4,7}$ (D) \\ Giacomo Spinato ${ }^{5,6}$
}

Published online: 6 March 2020

(C) Springer-Verlag GmbH Germany, part of Springer Nature 2020

\section{Correction to: Child's Nervous System (2020) 36:485-489 https://doi.org/10.1007/s00381-020-04514-9}

The original version of this article unfortunately contained an error. The author apologizes for having communicated an interchanged author's first and family names. Given in this article are the correct author names.

Publisher's note Springer Nature remains neutral with regard to jurisdictional claims in published maps and institutional affiliations.

The online version of the original article can be found at https://doi.org/ 10.1007/s00381-020-04514-9

Piero Pavone

ppavone@unict.it

Gino Amleto Pacino

odonto.ro@azisanrovigo.it

Salvatore Cocuzza

s.cocuzza@unict.it

Antonino Maniaci

tnmaniaci29@gmail.com

Maria Cristina Da Mosto

mariacristina.damosto@aulss2.veneto.it

Giacomo Spinato

spin.giacomo@gmail.com

1 Oral and Maxillo-Facial Department, Provincial Rovigo Hospital, Rovigo, Italy
2 Department of Medical and Surgical Sciences and Advanced Technologies "GF Ingrassia", ENT Section, University of Catania, Catania, Italy

3 Otorhinolaryngology Department, Treviso Hospital, University of Padova, Padua, Italy

4 Section of Pediatrics and Child Neuropsychiatry, Department of Clinical and Experimental Medicine, University of Catania, Catania, Italy

5 Department of Neurosciences, Section of Otolaryngology and Regional Centre for Head and Neck Cancer, University of Padova, Padua, Italy

6 Department of Surgery, Oncology and Gastroenterology, Section of Oncology and Immunology, University of Padova, Padua, Italy

7 Department of Pediatrics, University of Catania, 95100, Via Santa Sofia, Catania, Italy 